\title{
¿Selección de ojos o pacientes? Trampa estadística
}

\section{Selection of eyes or patients? Statistical trap}

\author{
Christian Fau ${ }^{1 *}$, Solange Nabzo ${ }^{1}$ y Veronica Nasabun ${ }^{2}$ \\ ${ }^{1}$ Fundación Oftalmológica 2020, Iberoamerican Cochrane Network; ${ }^{2}$ Universidad Andrés Bello, Escuela de Enfermería. Santiago, Chile
}

En los estudios clínicos o epidemiológicos en oftalmología, a diferencia de otras especialidades, se presenta un problema en la selección de la muestra, ya que los sujetos de estudio tienen potencialmente dos fuentes de datos interrelacionados, es decir, dos ojos para contribuir al estudio. Potencialmente podría ser reclutado un ojo o podríamos duplicar nuestra muestra reclutando ambos ojos, ya que si en un paciente hay información disponible en ambos ojos, ¿por qué no usarla?

El problema es que, probablemente, los hallazgos en el ojo izquierdo de un individuo sean generalmente más parecidos a los del ojo derecho del mismo individuo que a los del ojo de otro individuo. Esto se debe a múltiples factores, y se denomina correlación inter-ojo. Las técnicas estadísticas habituales se basan en la asunción de que los valores de las observaciones son independientes en cada ojo medido. En este caso, al incorporar los dos ojos, esto no se cumple. Actualmente, muchos artículos no tienen en cuenta este problema, y el ignorar este hecho puede conducir a un rechazo del estudio.

En la literatura hay principalmente 4 tipos diferentes de estudios en oftalmología, según los criterios de selección de la muestra ${ }^{1,2}:$ 1) diseño de sujeto, en el cual el objeto del análisis es el individuo, por ejemplo, estudios de estrabismo o de discapacidad visual; 2) diseño de ojos pareados, en el cual se incorporan ambos ojos y se compara uno contra el otro; 3 ) diseño de un ojo, en el cual se selecciona solo un ojo de cada paciente para ser incluido en el estudio, y 4) diseñe de dos ojos, en el cual algunos individuos contribuyencon un ojo y otros con dos, o todos con dos ojos, en este último caso, los dos ojos pueden ser, por ejemplo, aleatorizados a un mismo grupo de tratamiento, gruppos diferentes o mixtos.

Según el estudio de Lee, et al. ${ }^{1}$ se estima quẹ el $13 \%$ de los ensayos clínicos publicados corresponden a un diseño de sujeto o análisis de individuo los cuales no tienen grandes problemas en su disêño. Otro 13\% corresponde a un diseño de ojos pareados, que requiere de análisis estadísticos para datos@pareados, por lo cual tampoco presenta problemas metodológicos. Un $48 \%$ corresponde a un diseño dēojo único. Este es el diseño más usado, posiblemente porque es el método más simple, se utiliza la estadística clásica y no hay interferencia entre ojos. El principal problema de este método es que hay un potencial desperdicio de información que conduce a estimaciones menos precisas, ya que el otro ojo está disponible, muchas veces se mide y luego la iñformación no se utiliza y se desecha. En algunos estudios, en un intento por no perder la información segundo ojo, se utiliza un cálculo promedio de los dos ojos de aquellos pacientes que tienen los ôlos registros disponibles ${ }^{2}$. Además, en estos estudios hay que prestar especial atención a que se declare claramente qué método se utilizó para seleccionăr el Santiago, Metropolitana, Chile E-mail: chfauf@gmail.com (http://creativecommons.org/licenses/by-nc-nd/4.0/).
Fecha de recepción: 20-06-2019

Fecha de aceptación: 05-09-2019 DOI: 10.24875/RMO.M20000095

Disponible en internet: 02-01-2020 Rev Mex Oftalmol. 2020;94(1):53-54 www.rmo.com. $m x$

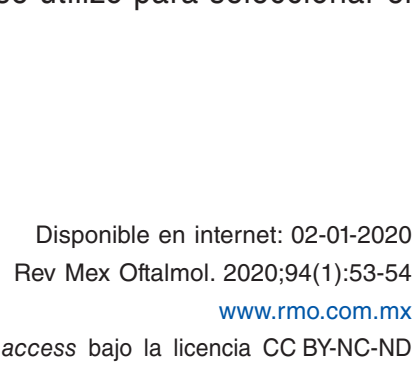


ojo incluido en el análisis. Lee ${ }^{1}$ estimó que, en el $52 \%$ de los artículos publicados, el método no se declara claramente. El método, además, puede ser sesgado si no se utilizó aleatorización, por ejemplo, uso del peor o mejor ojo, ojo operado, el primer ojo con enfermedad, solo ojos derechos o izquierdos, o en unos pacientes se aleatorizó y en otros se seleccionó el ojo que tenía la información completa. Por último, el diseño de dos ojos es el más complejo de todos los diseños. Lee estimó que el $19 \%$ de los estudios publicados corresponden a este diseño, en ninguno de los estudios analizados por él encontró que los autores ajustaran el tamaño muestral, y solo en el 39\% de los estudios los autores ajustaron el análisis estadístico por la correlación entre los ojos. En el estudio de Murdoch ${ }^{2}$ este valor incluso bajó a un $11 \%$.

Los análisis que no tienen en cuenta la correlación ocular suponen que tenemos más información de la que tenemos realmente. Esto lleva a un grado de precisión falsamente alto, una infraestimación del error estándar, los intervalos de confianza se vuelven más precisos y las diferencias entre los dos grupos generan valores de $p$ falsamente más pequeños de lo que realmente son $^{2,3}$. Si los autores no tienen posibilidades de realizar análisis estadísticos más complejos, tanto en el cálculo de tamaño muestral como en el ajuste de la correlación que ocurre en los resultados, deben evitar este tipo de diseño, ya que se arriesgan a que al enviar un artículo para evaluación a una revista este sea rechazado; o de ser aceptado, cuando este sea leído será descartado rápidamente por errores de validez interna y no generar ningún impacto como publicación.

\section{Conflicto de intereses}

Los autores declaran que no existen conflictos de interés.

\section{Responsabilidades éticas}

Protección de personas y animales. Los autores declaran que los procedimientos seguidos se conformaron a las normas éticas del comité de experimentación humana responsable y de acuerdo con la Asociación Médica Mundial y la Declaración de Helsinki:

Confidencialidad de los datos. Los autores declaran que han seguido los protocolos de su centró de trabajo sobre la publicación de datos de pacientes.

Derecho a la privacidad y consentimiento informado. Los autores han obtenido el consentimiento informado de los pacientes y/o sujetos referidos ê el artículo. Este documento obra en poder del autog̊ de correspondencia.

\section{Bibliografía}

1. Lee CF, Cheng ACO, Fong DYT. Eyes or Subjects: Are Ophthalmic Randomized Controlled Trials Properly Designed and Analyzed? Ophthalmology. 2012;119(4):869-72.

2. Murdoch IE, Morris SS, Cousens SN. People and eyes: statistical approaches in ophthalmology. Br J Ophthalmol. 1998;82(8):971-3.

3. Newcombe RG, Duff GR. Eyes or patients? Traps for the unwary in the statistical analysis of ophthalmological studies. Br J Ophthalmol. 1987;71(9):645-6. 